\title{
Deutlich weniger Migränetage durch CGRP-Antikörper
}

Fragstellung: Wie steht es um Wirksamkeit, Verträglichkeit und Sicherheit des monoklonalen Antikörpers Erenumab gegen den CGRP(Calcitonin gene related peptide)-Rezeptor in der Langzeitprophylaxe der episodischen Migräne?

Hintergrund: Etwa 30 \% aller Migränepatienten haben sehr häufige und schwere Migräneattacken, sodass eine prophylaktische Behandlung notwendig wird. Die erfolgt nach den Leitlinien der DGN und der Deutschen Migräne- und Kopfschmerzgesellschaft mit Betablockern, Antikonvulsiva wie Topiramat oder Valproinsäure, Flunarizin oder Amitriptylin. Leider sind Adhärenz und Compliance mit der medikamentösen Prophylaxe nicht sehr gut, was zum einen an der begrenzten Wirksamkeit, zum anderen an der Vielzahl unerwünschter Arzneimittelwirkungen liegt. Daher war es dringend notwendig, eine spezifische Migräneprophylaxe zu entwickeln, die bei zumindest identischer Wirksamkeit wie bei den klassischen Substanzen weniger Nebenwirkungen hat, und bei der Adhärenz und Compliance gewährleistet werden können. Vor diesem Hintergrund wurden monoklonale Antikörper gegen CGRP oder den CGRP-Rezeptor entwickelt, die in der Zwischenzeit Phase-II- und -III-Studien bei episodischer und chronischer Migräne durchlaufen haben. In der vorliegenden Publikation werden Langzeitergebnisse einer Migräneprophylaxe mit Erenumab (AMG334) berichtet.

Patienten und Methodik: Die Patienten wurden ursprünglich in eine zwölfwöchige doppelblinde, placebokontrollierte Studie eingeschlossen, in der sie ent-

Ashina M, Dodick D, Goadsby PJ et al. Erenumab (AMG 334) in episodic migraine: interim analysis of an ongoing open-label study. Neurology 2017; 89: $1237-43$ weder mit $10 \mathrm{mg}, 21 \mathrm{mg}$ oder $70 \mathrm{mg}$ Erenumab verglichen mit Placebo behandelt wurden. Erenumab wurde alle vier Wochen subkutan appliziert. Nach dem Ende der Doppelblindphase, die publi- ziert wurde [1], überführte man die Patienten in eine offene Extensionsphase, in der sie $50 \mathrm{mg}$ Erenumab alle vier Wochen erhielten. In einer präspezifierten Interimsanalyse wurden alle Patienten ausgewertet, die über ein Jahr behandelt worden waren. Die Änderung der Migränetage pro Monat sowie eine Reduktion der Migränehäufigkeit von mehr als $50 \%$, mehr als $75 \%$ und $100 \%$ (migränefrei) wurden erfasst.

Zur Lebensqualitätsmessung wurden der Headache Impact Test (HIT), der Migraine-Specific Quality of Life-Fragebogen, der Migraine-Disability-Assessment-Test (MIDAS) und Nebenwirkungen sowie schwerwiegende unerwünschte Arzneimittelwirkungen erfasst.

Ergebnisse: An der Studie nahmen ursprünglich 472 Patienten in einem mittleren Alter von 41 Jahren teil. $80 \%$ der Teilnehmer waren Frauen. Bei Eintritt in die Studie hatten die Patienten im Mittel neun Migränetage pro Monat. An der Langzeitstudie nahmen 383 Patienten teil, die über einen medianen Zeitraum von 575 Tagen behandelt wurden. Die mittlere Zahl der Migränetage betrug 8,8 bei Eintritt in die Studie, 6,3 Tage am Ende der Doppelblindphase und 3,7 Tage nach 64 Wochen. Nach 64 Wochen lag die Responderrate für eine Reduktion von mehr als $50 \%$ bei $65 \%$ und von mehr als $75 \%$ bei $42 \%$ der Studienteilnehmer. Migränefrei waren 26\% der Patienten. Bei allen Lebensqualitätsinstrumenten kam es zu einer signifikanten Reduktion in der doppelblinden Behandlungsphase, die bis zur Woche 64 anhielt. Es traten keine unerwarteten und unerwünschten Arzneimittelwirkungen auf und es gab keine Sicherheitsprobleme. Zwei vaskuläre Ereignisse, ein vaskulärer Todesfall und ein Myokardinfarkt, waren nicht mit der Studienmedikation assoziiert.

Schlussfolgerungen: Eine offene Langzeitstudie mit Erenumab bei Patienten mit episodischer Migräne zeigt eine lang anhaltende gute Wirkung, bei einem hervorragenden Verträglichkeitsprofil.

\section{- Kommentar von Hans-Christoph Diener, Essen}

\section{Anhaltend verbesserte Lebensqualität}

Bei allen neuen Medikamenten ist es wünschenswert und notwendig, dass jenseits der meist kurzen doppelblinden Behandlungsphase eine Langzeittherapie erfolgt, um zu sehen, ob die Therapieeffekte anhalten, und ob es auf längere Sicht Probleme mit der Verträglichkeit oder Sicherheit gibt. Beides ist in der offenen Beobachtungsstudie von 338 Patienten, die $70 \mathrm{mg}$ Erenumab alle vier Wochen subkutan erhielten, nicht der Fall. Die Reduktion von Migräneattacken beziehungsweise Migränetagen pro Monat wurde im Beobachtungszeitraum geringer. Die Lebensqualität verbesserte sich anhal- tend, und besonders wichtig war, dass keine unerwünschten Arzneimittelwirkungen beobachtet wurden. Da Erenumab subkutan appliziert wird, sind auch Adhärenz und Compliance gewährleistet. Es bleibt jetzt abzuwarten, ob zu den anderen CGRP-Antagonisten ähnliche Langzeitergebnisse publiziert werden können.

\footnotetext{
Referenz:

1. Tepper S et al. Lancet Neurol 2017; 16: 425 - 34
} 